\title{
Integration of Modelica models into an existing simulation software using FMI for Co-Simulation
}

\author{
Matthias Pazold ${ }^{1}$, Sebastian Burhenne ${ }^{2}$, Jan Radon ${ }^{3}$, Sebastian Herkel ${ }^{2}$ and Florian Antretter ${ }^{1}$ \\ ${ }^{1}$ Fraunhofer Institute for Building Physics, Holzkirchen, Germany \\ ${ }^{2}$ Fraunhofer Institute for Solar Energy Systems, Freiburg, Germany \\ ${ }^{3}$ Agr. University of Cracow, Poland \\ Matthias.Pazold@ibp.fraunhofer.de
}

\begin{abstract}
The Functional Mock-up Interface (FMI) opens new opportunities for the development and extension of existing non-Modelica simulation programs with Modelica models. For the developer this is a productive way to design and validate new complex simulation models with multi-domain modeling languages such as Modelica. With the standardized Functional Mock-up Interface (FMI) and the Functional Mockup Unit (FMU) export it is possible to execute these models within other software tools, including information exchange during the simulation. However, there are some design requirements in Modelica, which have to be taken into account. In this paper, models for different HVAC (Heating, Ventilation and Air Conditioning) equipment configurations are integrated into existing software using the FMI. An interface extension plug-in is developed to pick a specific FMU and execute it alongside the existing simulation algorithm. Two different coupling algorithms were investigated: the iterative and the cosimulation approach. Some issues and practical hints for a successful coupling and simulation are presented.

Keywords: Building Simulation; FMI for CoSimulation; HVAC
\end{abstract}

\section{Introduction}

The application of building performance software during the design process is standard in the design of energy efficient buildings. There are tools that solve the coupled heat and moisture transport in building components to avoid moisture related problems such as mold growth or rotting components. Different kinds of components (e.g. walls, windows, roof) are combined to a whole building model. Additionally, climate data and inner sources lead to a whole building envelope simulation software, which allows for an accurate assessment of the indoor environment and the energy consumption of the building. The WUFI ${ }^{\circledR}$ Plus software offers the possibility for such a simulation. Until now, the HVAC equipment was considered as an ideal heating and cooling system. Current activities aim to implement realistic models into WUFI ${ }^{\circledR}$ Plus to simulate HVAC systems. These models are written in Modelica [1]. The building envelope and the HVAC system influence each other significantly. This makes a separate simulation of both systems inaccurate and introduces special requirements for combining both in a co-simulation. The decision to implement the Modelica models into the existing software rather than model the building envelope with Modelica was made because of the big user community, which is familiar with the existing GUI and other user specific requirements. A possible way to include Modelica models into an existing building simulation program is the Functional Mockup Interface for Co-Simulation. The integration is described in this paper.

\section{Existing Software / Models}

\subsection{Building model}

WUFI ${ }^{\circledR}$ Plus is a holistic model based on the hygrothermal envelope calculation model developed by Künzel [2]. The hygrothermal behavior of the building envelope affects the overall performance of a building. WUFI ${ }^{\circledR}$ Plus is a building performance simulation tool, which computes the coupled heat and moisture transfer in the building components. These components are combined to a whole building model. Moisture sources or sinks inside the rooms or components, input from the envelope due to capillary 
action, diffusion and vapor ab- and desorption as a response to the exterior and interior climate conditions as well as the thermal parameters are taken into account. A stable and efficient numerical solver had been designed for the solution of the coupled and highly nonlinear equations. The conductive heat and enthalpy flow by vapor diffusion with phase changes in the energy equation are strongly dependent on the moisture fields. The vapor flow is simultaneously governed by the temperature and moisture field due to the exponential changes of the saturation vapor pressure with temperature. The differential equations are discretized by means of an implicit finite volume method. The model was validated by comparing its simulation results with the measured data of extensive field experiments [3]. The user can define design conditions for the indoor climate by setting minimal and maximal values.. To simulate the indoor climate, the software calculates heat and moisture balances for one or more building zones, regarding all the sources, sinks and transfers. So long as these balances are not satisfied during a time step, the interior temperature and humidity is adapted. For example, if the heat loss through the building envelope and ventilation is more than the solar and internal heat gains plus space heating capability, the interior temperature is decreased as long as the loss and the gain is equal.

\subsection{Modelica HVAC models}

The aim was to create simple but realistic HVAC models, which can be used by practitioners. This means that only necessary and obtainable plant information is required for these simulations. The computation time to simulate a building should not increase to times which are no longer acceptable for practitioners.

Systems to be simulated include:

- Condensing gas boiler

- Solar thermal collector

- Combined heat and power plants

- Heat pumps

- Bore hole heat exchangers

- Thermally activated building systems (TABS)

- Radiators

- Storage tanks

- Control equipment

- PV systems
The model development was done with the software Dymola 2012 [4]. To deliver realistic and validated plant equipment models, the above mentioned submodels are merged to complete HVAC configurations, an example is shown in Figure 1. This was done to increase the usability and avoid the risk of non-feasible configurations. In the end, the user chooses one HVAC configuration and has to select only a few necessary parameters or import them from a database.

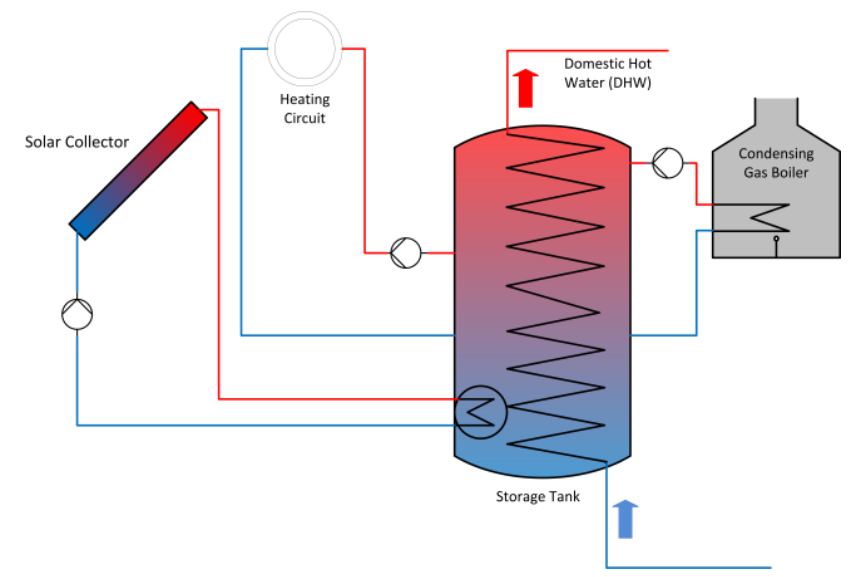

Figure 1: Exemplary HVAC configuration in WUFI ${ }^{\circledR}$ Plus

\section{Integration}

The first investigated coupling approach was to use Dymola specific export possibilities (Source Code generation). More details on this can be found in [5]. Finally the coupling with the Functional Mock-up Interface for Co-Simulation was chosen because of its unified convention and possibilities to perform the co-simulation. Merging the existing software and the Modelica HVAC models using the Functional Mock-up Interface for Model Exchange would require the development of a new solver for WUFI ${ }^{\circledR}$ Plus. Therefore, one of the main advantages in the context of the described application was that a solver is included in the FMU for Co-Simulation. In the described case it is the CVODE solver included in the Sundials solver package [6]. The selected solver within Dymola has no influence on the exported solver. The standardized interface provides some methods to interact with the model. Beside instantiating, initializing, setting and obtaining of values of defined variables and parameters there is the possibility to execute single time steps. Furthermore, there is a distinction between time varying variables and parameters. The value of parameters can be set before initializing the model; the value of variables can be set between the time steps. But these time 
varying variables must be declared as input within the Modelica code. Their causality must be set to Input. If parameters appear in if-statements in submodels, the model must be re-compiled for a change of their value. In the exported and compiled FMU such a parameter is automatically set to constant and the value is firmly anchored. Changing the value of constants within the FMU is not possible. If-statements are often responsible for discontinuities and events. They should be avoided during the model design process because they increase the computation time [7]. However, to set a parameter of an if-statement in the compiled FMU, a workaround is to define the parameter as input.

There are more than one HVAC configurations with different devices and different parameters and, consequently, many FMUs. WUFI ${ }^{\circledR}$ Plus has to interact with the HVAC system configuration, which is chosen by the user of the software. A FMU adapter (Figure 2) is written in the object-oriented language $\mathrm{C}++$ to manage dynamic FMU instantiation, initialization, set inputs, obtain outputs and execute time steps. Therefore, the adapter receives information about the different kinds of configurations and their parameters (their value references).

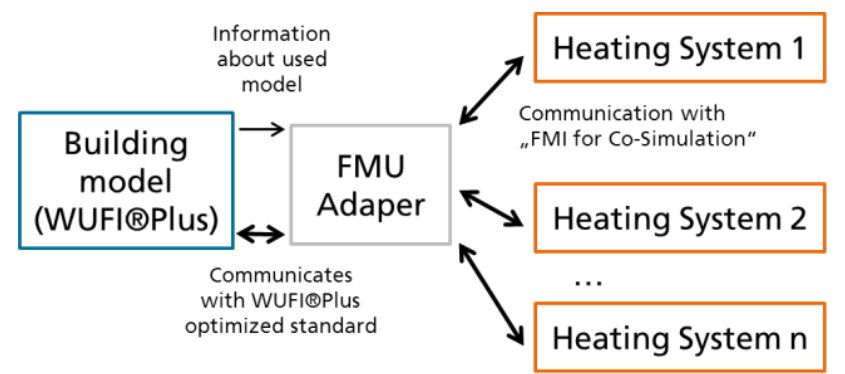

Figure 2: Communication between building model and heating systems

As mentioned, the building model and the HVAC models have to interact with each other. Some results of one are needed as input for the other. Two different insertion algorithms were investigated and are discussed below.

\subsection{Iterative approach}

As described before, WUFI ${ }^{\circledR}$ Plus uses an iterative process to simulate the interior temperature and moisture for defined zones. Also airflow is calculated iteratively. For short computation times there is a solver designed for fast convergence of these values with only a few iterations. Indeed, the HVAC systems influence the indoor climate. The first approach was to use the existing heat and moisture balance algorithm. The HVAC system receives, for example, the indoor set point temperature and the actual temperature of a zone and a time step and delivers the possible heat flow to the zone. If the heat balance is not satisfied, the current temperature will be in- or decreased and the HVAC system must iterate (Figure 3).

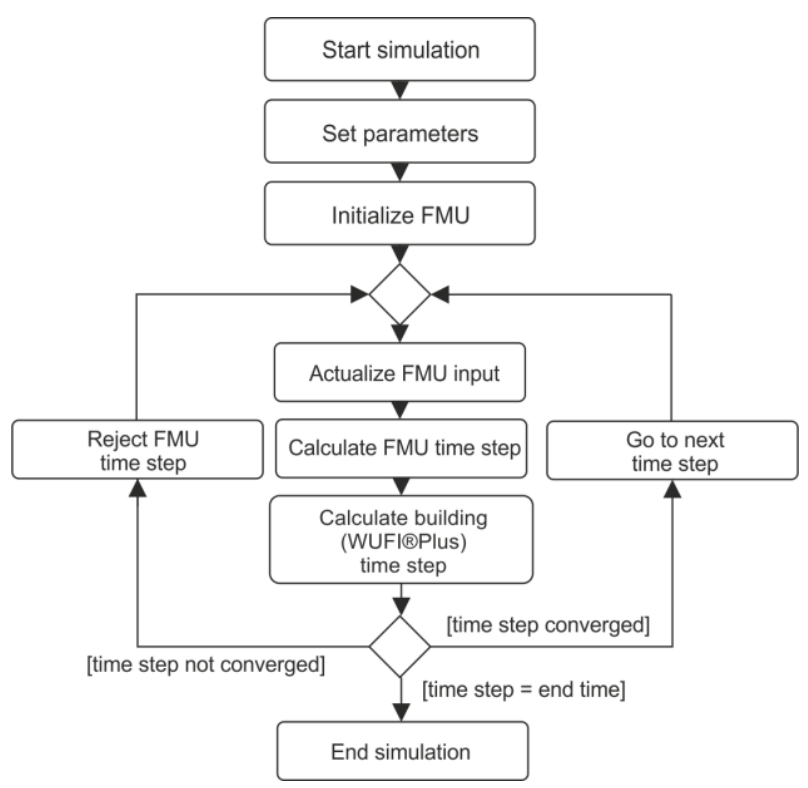

Figure 3: Flow chart - iterative approach

The advantage of this approach is to use the established flexible balance system. The HVAC model can be coupled in a fast way with only a few modifications of the WUFI ${ }^{\circledR}$ Plus algorithm. However, this method requires repeating and discarding of FMU time steps. Therefore the parameter newstep of fmidostep (..) can be set to fmiFalse if the capability flag canRejectsteps of the FMU is true. Until now this feature is not supported by the exported FMU. This is specific to Dymola and might not be the case for other simulation environments. However, in the analyzed case the missing feature is a problem for the implementation of the iterative approach.

If a time step is regarded as an entire simulation, a workaround could be to re-initialize the FMU for every time and iteration step. In order to retain all information, all time varying variables must be stored after a step and re-stored as initialization values for the next step. To repeat a step, the values of the last step are used. This could be time and memory consuming. Furthermore, some states of the model, which cannot be stored in the cache, may change during a time step.

A further issue of this coupling approach is that the iteration might end in a continuous loop. The heat supply system models are designed to deliver a heat 
flow to the room, when the current room temperature is lower than the set point temperature. This is implemented using a thermostatic valve model. The building model iterates the room temperature with the heat balance, including the heating system as a kind of heat source. If the balance is positive, the room temperature can be increased for the next iteration step. In this case, the current room temperature might become equal or greater than the set point temperature and in return the heating system model calculates no heat gain. If the heat balance ends with a negative sum, the room temperature is decreased. The heat supply system reconvenes a heat gain for the next iteration. This leads to a continuous loop.

\subsection{Co-simulation approach}

The mentioned issues with the iterative approach lead to a real co-simulation approach. The iterative process has been removed, so there is no requirement to repeat time steps within the FMU. Therefore the building envelope model (WUFI ${ }^{\circledR}$ Plus) and the HVAC model calculate the steps alternately with a ping-pong method. A usual simulation time step, to simulate a whole year, is one hour. For the alternately co-simulation this time step size, with ,e.g. constant room temperatures, very likely leads to unrealistic simulation results. One physically realistic solution is to decrease the time step size.

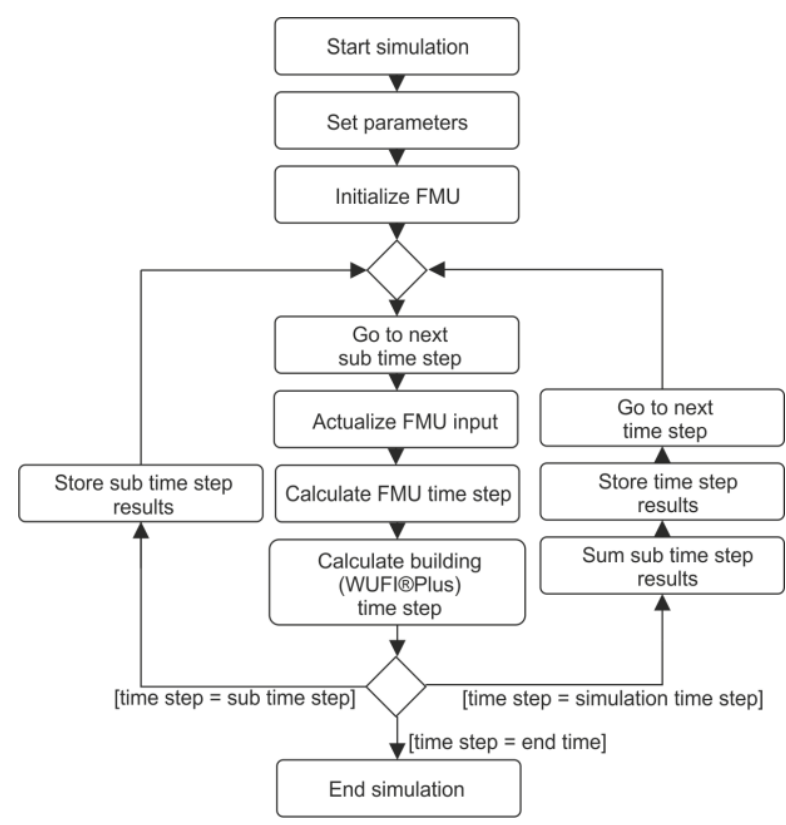

Figure 4: Flow chart - co-simulation approach

The explicit algorithm (Figure 4) is the following, described with thermal values: The plant equipment model calculates a few seconds with a constant interior temperature. Dependent on the heat emitting system (e.g. radiator) and its heat capacity and performance, this is possible because of the fast response time of the active HVAC system. The result is still the heat flow which is added as heat gain to the building zones. Simultaneously, the building is simulated with the last heat gain. Depending on the heat balance the new interior temperatures for the next time step are calculated. With this method, small time steps depending on the time constant of the heating system must be used (e.g., five seconds). This leads to increased computation times. However, these small sub time steps must not be stored in the results. Furthermore, the building model converges faster with small time steps, which saves some computation time.

First tests with a time step of five seconds showed an increased demand of simulation time of about one third compared to the WUFI ${ }^{\circledR}$ Plus simulation without the HVAC models. The results of a Dymola simulation compared to the results of an external FMU simulation of the HVAC system are equal.

\section{Conclusions}

The multi-zone building model and the HVAC models are complex models with a lot of variables and their own specialized solver. Separately they are proven, validated and stable for many kinds of simulations. The described weak coupling using the cosimulation approach seems to be a reasonable technique. Exported FMUs, acting as sub-models with defined in- and output, can be used to supplement the building model. The authors believe, that in principle, the merging of the models is possible with the introduced iterative and co-simulation approach. However, not supported features of the exported FMU make the iterative approach unfeasible. A still acceptable computation time with the co-simulation approach led us to the conclusion that this is a more suitable approach in the described case. The sum of heat gains over the sub time steps delivered by the HVAC configuration is realistic. However, future work will include more investigations about the chosen time intervals and the handling of discontinuous input.

\section{Acknowledgement}

This study was funded by the German Federal Ministry of Economics and Technology (BMWi 0329663L) 


\section{References}

[1] Elmqvist, Hilding 1997. "Modelica - A unified object- oriented language for physical systems modeling." Simulation Practice and Theory 5, no. 6., 1997.

[2] Künzel, H. M. 1994. Simultaneous Heat and Moisture Transport in Building Components. Dissertation. University of Stuttgart, Download: www.building-physics.com

[3] Lengsfeld, K.; Holm, A. 2007. Entwicklung und Validierung einer hygrothermischen Raumklima-Simulationssoftware WUFI ${ }^{\circledR}$ Plus, Bauphysik 29 (2007), Heft 3, Ernst \& Sohn Verlag für Architektur und technische Wissenschaften GmbH \& Co. KG, Berlin.

[4] Dassault Sysèmes AB 2011. Dymola. Dynamic Modeling Laboratory. Dymola Release notes, Lund, Sweden.

[5] Burhenne, S.; Radon, J.; Pazold, M.; Herkel, S.; Antretter, F. 2011. Integration of HVAC Models into a Hygrothermal Whole Building Simulation Tool, Proceedings of Building Simulation 2011: 12th Conference of International Building Performance Simulation Association, Sydney, Australia.

[6] Hindmarsh A. C.; Brown P. N.; Grant K. E.; Lee S. L.; Serban R.; Shumaker D. E.; Woodward C. S. 2005. SUNDIALS: Suite of Nonlinear and Differential/Algebraic Equation Solvers, ACM Transactions on Mathematical Software, 31(3), pp. 363-396, 2005. Also available as LLNL technical report UCRL-JP-200037.

[7] Felgner, F.; Liu, L.; Frey G. June 2011. Vergleich numerischer Löser zur Simulation steifer und hybrider Systeme. Proceedings of the Kongress Automation 2011, VDIBerichte 2143, Baden-Baden, Germany, pp. 303-306 (extended 12-pages paper on CD), June 2011. 
